\title{
"Los códigos en la construcción en Colombia: Aproximaciones y aplicaciones”
}

\author{
"Building Codes in Colombia: Approximations and Implementation”
}

\author{
Hernando Vargas Caicedo ${ }^{(1)}$ \\ Editor invitado \\ (1) M.of Sc. Architectural Studies. M.C.P Master of City Planning. Profesor Titular, Departamento de Ingeniería Civil y Ambiental y \\ Departamento de Arquitectura. Universidad de los Andes, Bogotá, Colombia. hvargas@uniandes.edu.co
}

DOI: http://dx.doi.org/10.16924/riua.v0i41.784

Como expresión de la preocupación ciudadana y académica por el desarrollo y el futuro en la aplicación de códigos de la edificación, la Revista de Ingeniería convocó en 2014 al Foro "Los códigos en la construcción en Colombia: Aproximaciones y aplicaciones”. Allí se recogieron experiencias internacionales de Europa, Norteamérica y Latinoamérica así como análisis de recientes experiencias colombianas a nivel nacional y local. El dossier demuestra la variedad existente de tendencias normativas y de situaciones de aplicación a sus entornos y enumera acciones de las agendas investigativa, normativa, formativa y de gobierno que se derivan de las amplias consecuencias de los códigos como factores del bien público.

Eduardo Behrentz, decano de la Facultad de Ingeniería, presentó la temática del evento como relevante frente a la seguridad y bienestar generales, con consecuencias sobre el patrimonio de las personas y la credibilidad de actores e instituciones competentes. Por esto, destacó que es necesario identificar las prácticas inapropiadas a través de análisis exigentes para proponer soluciones estratégicas y objetivas. Hizo hincapié en el caso de las curadurías urbanas en Colombia sobre las cuales se han levantado expectativas muchas veces incongruentes alrededor del alcance que pueden cumplir en las tareas de control. Insistió en que no deberían complejizarse procesos de control sin tomar en cuenta rasgos del entorno específico local y que habría de examinarse la arquitectura institucional y normativa. Estos esfuerzos resultan inútiles si no se ofrece educación técnica y compromiso ético sobre la responsabilidad profesional a través de los programas académicos, más allá del refinamiento de los sistemas de auditoría.

Como vocero del Ministerio de Vivienda, Alonso Cárdenas planteó las iniciativas principales que esa institución promueve actualmente en materia de codificación de la construcción. Aparte de su tarea misional frente a la actualización de la normativa sobre construcción sismo resistente, para fortalecer los sistemas regulatorios en vivienda y desarrollo urbano, se han considerado disposiciones relativas a uso eficiente de agua y energía. Para controlar la actividad constructora y la de los licenciadores urbanísticos, se estudian pólizas que protejan a compradores de vivienda nueva, fondos para apoyar reconstrucciones, nuevo enfoque en la supervisión técnica de la construcción, establecimiento de registro nacional profesional relativo a diseñadores, revisores y supervisores de obras, reglamentaciones sobre los certificados de ocupación y control sobre las curadurías urbanas.

David Walls, del International Code Council (ICC), expuso el origen de esa organización que, en años recientes, ha venido a integrar nacionalmente codificaciones sobre edificación en los Estados Unidos y diseminándolas internacionalmente en áreas de África, Asia y América. Está formada por miembros privados de la industria de la edificación norteamericana y, desarrolla códigos modelo, que son adaptados y adoptados a niveles locales; ofrece una variedad de materiales y servicios en materia de documentación regulatoria, formación, certificación, verificación, evaluación de productos y acreditación. Es claro que actualmente no solamente se busca edificación segura sino sostenible. A partir de los primeros códigos que fueran impulsados por compañías de seguros como partes afectadas por eventos de incendio, sismos y daños, desde 1994 se fue dando la esperada integración en Norteamérica de sus grandes códigos regionales. De esta forma, se ha consolidado una amplia organización autofinanciada que cuenta con planta técnica para su operación. Finalmente, son las organizaciones gubernamentales las que, después de la invitación pública a los interesados en procesos normativos, deciden en forma de consenso sobre la adopción de normativas. De esta manera, se completa un conjunto de quince códigos temáticos que cubren desde capítulos tradicionales de las reglamentaciones de sistemas de la edificación hasta el enfoque actual de construcción sostenible. Los códigos buscan garantizar seguridad, salud y bienestar públicos en el ambiente edificado fijando requisitos mínimos frente a riesgos particulares para la seguridad vital y el patrimonio por agentes como el fuego y otras amenazas. Más allá de la 
seguridad estructural, abordan medios de evacuación, condiciones sanitarias, luz y ventilación y conservación de energía.

Para presentar la experiencia española en la adopción del nuevo código técnico de edificaciones de 2006, Javier Serra y José Antonio Tenorio, explicaron el enfoque de prestaciones o desempeño de nuevas familias de códigos que se proponen superar la naturaleza prescriptiva, considerada rígida y cerrada frente a la innovación. De esta forma, al enunciar objetivos y no medios, se facilita que, una vez definidas las características de las edificaciones para los usos que deben cumplir, se ordenen los niveles normativos en jerarquía descendiente señalando sucesivamente principios sociales, requisitos funcionales, métodos de verificación y conjuntos de soluciones aceptables. Se destacó cómo esta filosofía se incubó en Europa desde 1953 y se apoyó en las necesidades de mercados ampliados de materiales, componentes y sistemas constructivos. Conforme a la ley española de ordenación de la construcción de 1999, para los códigos prestacionales se tienen grupos temáticos, referidos a los grandes conjuntos: la funcionalidad, la utilización, la accesibilidad y el acceso a los servicios de telecomunicación; los aspectos de seguridad estructural, en caso de incendio, y en utilización; los requisitos relativos a la habitabilidad relativos a higiene, salud y protección del medio ambiente. La actualización permanente del código español cuenta con sistemas de información pública a sus usuarios y apoyos como catálogo de elementos constructivos en prácticas adecuadas al clima y formas constructivas locales.

En la presentación de Luis Enrique García, se hizo un recuento de antecedentes principales de la normativa de construcciones sismo resistentes en Colombia y de hitos que ha tenido dicha codificación desde su primera expedición en 1984. Se destacó la naturaleza de los códigos como forma de contrato social, que busca favorecer el ejercicio legal de las actividades de

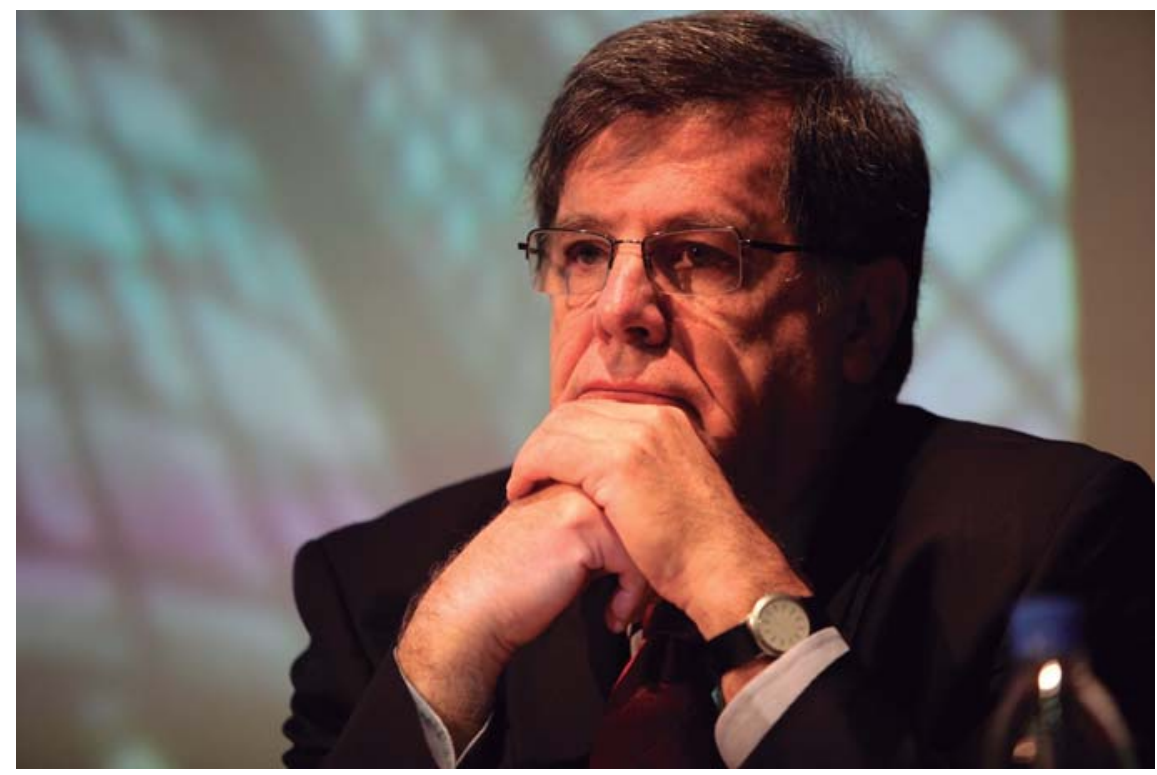

Hernando Vargas Caicedo. Fuente: Archivo Particular, Universidad de los Andes

edificación. Se advirtió también que existen deficiencias en las formas de práctica cuando se reducen los diseños a planos, descuidando el papel de las especificaciones. Se planteó, además, que anteriormente a los códigos se les daban prácticas cuidadosas, considerando seriamente las características de diseño, más allá de cumplir unos mínimos. Para el caso colombiano, se lamentó que por carencia de coordinaciones, se dieran en paralelo leyes sobre ordenamiento territorial y construcción sismo resistente que no articularon elementos de control necesario para la aplicación normativa. Por otra parte, se advirtió que, por la posición del país sobre la confluencia de grandes placas tectónicas, la sismicidad es relevante con experiencia acumulada en diversos eventos y seguida mediante red nacional de acelerógrafos para registrar en forma continua esos fenómenos. Se señaló que las disposiciones de codificación han sido adoptadas en condiciones de excepción y cómo, a partir de propuestas normativas inicialmente desarrolladas en los Estados Unidos, se han propuesto, ajustado y adoptado gradualmente reglamentaciones para la construcción sismo resistente en el país, incorporando temáticas adicionales a la inicial de estructuras sin que, aún, se incluyan prescripciones de tipo ambiental en las disposiciones.

José Pedro Campos expuso los grandes cambios cumplidos en la organización de los procesos normativos de construcción en Chile en las dos décadas pasadas. A partir de determinantes como la ley de calidad de 1996 y el terremoto y maremoto de 2010, se han dado modificaciones sustanciales legales, reglamentarias y normativas de la construcción. Una de ellas fue el establecimiento del Instituto de la Construcción como ente técnico de composición transversal que abarca participación pública y privada, involucrando a los ministerios, las constructoras, las inmobiliarias, las industrias, los colegios profesionales, la academia y las universidades. Dentro de sus misiones hay tareas notables alrededor de metas sobre construcción sustentable y eficiencia energética. Como parte del nuevo conjunto normativo que ha resultado de su acción promotora y coordinadora, se han dado normas de carácter general, de diseño arquitectónico, de estructuras, acondicionamiento ambiental, seguridad, materiales y componentes, instalaciones, herramientas, equipos y mobiliario. Son destacables eventos como la ley de 2002 que hace obligatoria la revisión del proyecto 
de cálculo estructural para algunos tipos de edificación y la presencia dentro del Instituto del Consejo Nacional de Normalización de la Construcción - CNNC. Igualmente, se enfatizó que respecto de la vivienda se ha implementado un programa en etapas para aislamiento térmico en componentes y zonificación térmica. Es esencial el papel de la primera ley de la calidad de la construcción que establece la responsabilidad de la calidad de la obra por parte del propietario primer vendedor, para enfrentar la anterior dispersión de responsabilidades entre diseñadores, constructores y supervisores. Con la tercera ley de calidad se han exigido inspectores técnicos de obra. Por otro lado, advierte varias condiciones por cuidar en los procesos normativos, entre las que destaca reglamentar oportunamente las nuevas leyes, modernizar el procedimiento de juicio civil, conformar las OSEN u organizaciones sectoriales para estudio de normas, proveer recursos públicos para el desarrollo normativo, revisar los seguros asociados a la construcción, mejorar la coordinación entre entidades que elaboran normativas y regulan la construcción e incorporar nuevos estándares, materiales, sistemas y productos.

Camilo Villate y Clemencia Escallón plantearon sintéticamente el ejercicio que un equipo de la Universidad de los Andes cumplió en 2010 para estudiar las actualizaciones que el Código de Construcción de Bogotá, adoptado en 1995, debería tener para ajustarlo a la condición de la sostenibilidad. Con una revisión internacional de códigos y estándares, se establecieron referencias sobre tendencias normativas. Aunque la Universidad de los Andes inició su participación en el Código de Construcción de Bogotá desde 1985, cuando desde las entidades del Distrito la convocaron para elaborar el primer documento finalmente aprobado por el Concejo Distrital por el Acuerdo 20 de 1995, dicha normativa, aunque vigente legalmente, ha tenido escaso conocimiento y aplicación. Con el enfoque de sostenibilidad y apoyo de secretarías distritales, el equipo de varias disciplinas planteó reformular el código, basado anteriormente en enfoque prescriptivo, conservando sus temáticas principales sobre diseño arquitectónico, instalaciones, licencias, consideraciones de construcción y de ocupación. Como marco general, se consideraron la seguridad, la salubridad y el bienestar de la comunidad y se advirtió que, en el caso de Bogotá, el POT vigente se fundamenta de manera adecuada en la estructura ecológica principal. A partir del mismo, como estrategia de ocupación territorial, se debería conectar una escala intermedia, correspondiente a un código de urbanismo, para finalmente definir el nuevo código de construcción, del paramento predial hacia su interior, con el enfoque de sostenibilidad. En la filosofía de respeto por los semejantes, el medio ambiente y los materiales, el código debe considerar el ciclo de vida de las edificaciones hasta su disposición final. La información estructurada para su nueva configuración se detalló en fichas y acciones específicas, que en su conjunto, serían parte del código de construcción. Se llamó la atención sobre la necesidad de unificar y alinear el código local con los de carácter nacional y local de mayor entidad y de usarlo como base para la política de construcción sostenible y el eco-urbanismo, temas importantes que pretende promover la Secretaría de Ambiente en el Estándar Único de Construcciones Sostenibles para el Distrito Capital.

Por último, Diego Restrepo del Programa Gerencia de Vivienda Segura de Medellín, explicó el caso de esa ciudad en materia de control de construcción y control urbano y las acciones de la alcaldía para la década iniciada en 2014. Señaló el desafío que representa la alta proporción de informalidad en la edificación urbana, en ocasiones resultante de acciones de promoción de la vivienda de crecimiento progresivo. Se plantea que existe un problema sistémico, que podría tener alcance nacional y que demanda que, después de la protección integral de la vida, se cuiden el patrimonio y el derecho a la vivienda, para evitar que se confundan responsabilidades por deficiencias en control municipal urbanístico y de construcciones. El caso del colapso de la torre 6 del conjunto Space evidenció diseños estructurales que no cumplían las normativas, ausencias o deficiencias en las revisiones de memorias y planos estructurales y, conforme a los informes técnicos sobre causas incidentes en su caída, deficiencias en resistencia de materiales, inexistencia de supervisión técnica adecuada y respuesta negligente ante señales de sobreesfuerzos en la estructura. A juicio del expositor, ante los vacíos legales relativos al ámbito de controles urbanísticos y constructivos, debe reglamentarse la revisión de diseños y crearse control de curadurías y de la actividad constructora e inmobiliaria. Se requieren acciones sumadas como supervisiones técnicas e interventorías externas, certificado del permiso de ocupación de la obra, utilización de bitácora de obra, seguros obligatorios para vivienda segura. En programas de largo plazo, como el que se propone Medellín, se considera incorporar al nuevo Plan de Ordenamiento Territorial la seguridad de vivienda y de edificaciones y redefinir el control urbano más adecuado para asegurar el cumplimiento normativo. 